\title{
A Business and Solution Building Block Approach to EA Project Planning
}

\author{
Graham McLeod \\ Inspired, Cape Town, South Africa \\ University of Cape Town, South Africa \\ grahamainspired.org
}

\begin{abstract}
Many EA groups battle to establish an overall programme plan in a way that is integrated, achievable and understandable to the stakeholder and sponsor community as well as the downstream implementation groups, including: IT, Process Management, Human Resources and Product Management. This paper presents an approach that achieves these objectives in a simple way. The approach is currently being implemented in a fairly new enterprise architecture function within an aggressively expanding Telco with promising results. The problem is introduced and a solution including meta model and visual representations is discussed. Early findings are made to the effect that the technique is simple to apply as well as being effective in establishing shared understanding between the EA function, project sponsors, project stakeholders and IT personnel. The technique is explicated with an example that should make it easy for others to replicate in their own setting.
\end{abstract}

Keywords: Enterprise Architecture, Building Blocks, Project Scope, Planning, TOGAF, Inspired EA Frameworks.

\section{Introduction to the Problem}

The author and colleagues are engaged in a consulting capacity with a rapidly expanding Telco in South Africa. The organization has a newly established enterprise architecture (EA) function. EA is gaining good traction and driving several themes, including: building the EA capability and governance; implementing an ambitious five year growth strategy; providing architectural oversight to active projects and supporting a collection of projects focussed on improvements to the core value chain. A traditional value chain approach, ala Porter, has been employed [1]. A blend of the Inspired [2-3] and TOGAF [4] EA methods and frameworks is being used, with the emphasis on the former. This paper describes a situation and solution relative to the "enhancement of core value chain" projects. The solution will in time be more broadly applied in the strategic theme as well. We believe the approach has general applicability for EA teams in other organizations and settings.

The situation relative to the core value chain involved a number of projects which had already begun, prior to the architecture oversight. Two prominent ones were Quoting and Billing. The core value chain and their position in this can be seen in Figure 1. 
The Quote and Bill value chain elements were being addressed by active projects at the time of this intervention. Note: The value chain model did not initially include the support capabilities, viz. Product Management and Data and Information Management. The latter was also an active (but early stage) project in the environment. Product Management was a capability supported by a business department. Both the Quote and Billing projects highlighted the need for more capable, flexible, integrated and consistent Product Management, leading to it being identified as a focus area and the creation of a new project to address the related requirements. The evolution of the models and approach is described in the following sections.

\section{Problems Identified}

Problems in stakeholder expectations, sponsor communication and development project alignment were highlighted by a steering body meeting which reviewed the Quotations project. It emerged that there was little consensus upon the scope of the project, the dependencies between various elements and the release plan. Stakeholders and the sponsor were unclear what would be delivered, in what tranches and when. The EA function was asked to audit the project and make recommendations.

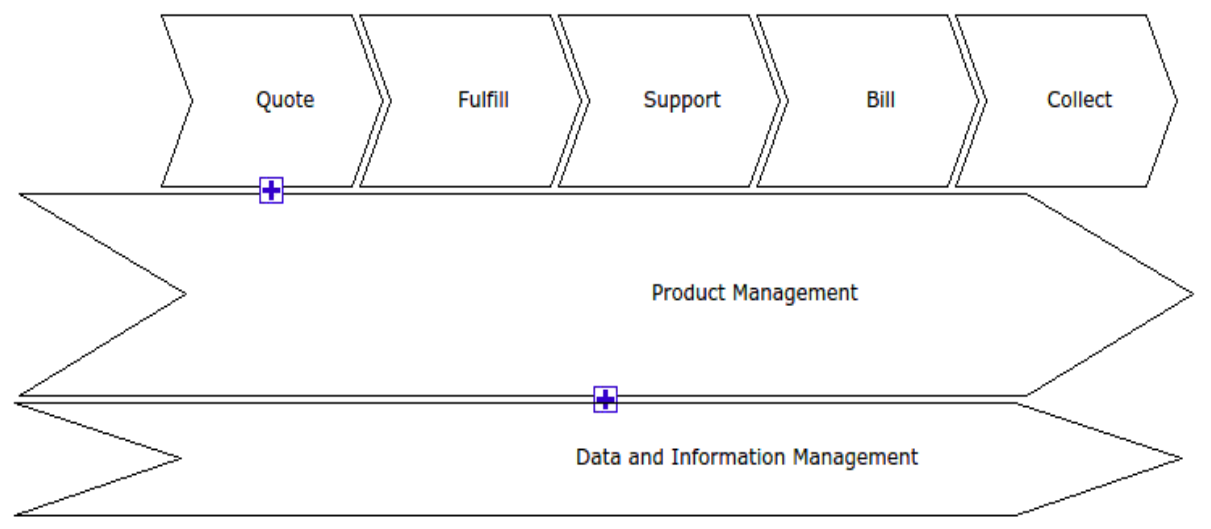

Fig. 1. Core value chain and support capabilities

The audit found that there was a traditional Business Requirements Specification (BRS) which had been done by a business analyst after extensive interviews with personnel in the line functions affected. The BRS effectively sketched an "end state" for the complete quoting automation, including embedded capabilities. The latter included product management, customer information, existing install base information and document generation capabilities. The scope of the BRS was thus large. It was not broken into any releases or delivery packages. The BRS had a tentative solution design, but this was not comprehensive. The major contribution of the BRS was the documentation of the business requirements at a logical level.

The development team in IT, meanwhile, was pursuing an agile project management approach [5-7], with a backlog of requirements managed in a tool. This 
detailed requirements at a more fine grained level and the team had identified some delivery tranches, which were variously dubbed versions and releases in discussions. The information was not in a form that could be easily packaged for consumption by the sponsor, stakeholders, EA or program management.

Programme management had visibility of the project via the previously mentioned steering body reviews. The discussions were ineffective as the terminology between releases and versions was not standardised, the agile requirements were not visible, and the allocation of capabilities between versions/releases was fluid. The problems could be summarised as follows: scope of requirements not clear; decomposition into releases not clear; definition of release and version terminology not standardised; lack of agreement between stakeholder expectations and project team plan; lack of visibility of capabilities to be delivered, dependencies and timing at program and project management levels; duplication of effort across projects; lack of traceability of requirements from traditional BRS to agile environment; confusion between requirements and solution elements; unclear link between value chain and supporting projects.

\section{Towards a Solution}

The audit was followed by a facilitated session where we teased out the capabilities contained in the BRS and the agile requirements at a business level and defined these as inter-related business building blocks (BBBs). TOGAF [4 section 37.2] recommends using building blocks to define required components of architecture (Architecture Building Blocks) and solution (Solution Building Blocks). TOGAF has a somewhat schizophrenic definition of building blocks: on one hand defining them thus:

"Architecture Building Blocks (ABBs) typically describe required capability and shape the specification of Solution Building Blocks (SBBs). For example, a customer services capability may be required within an enterprise, supported by many SBBs, such as processes, data, and application software.

Solution Building Blocks (SBBs) represent components that will be used to implement the required capability. For example, a network is a building block that can be described through complementary artifacts and then put to use to realize solutions for the enterprise. "“

but also at another point [section 33.2] defining them as the elements in the content or meta model:

"The content metamodel provides a definition of all the types of building blocks that may exist within an architecture, showing how these building blocks can be described and related to one another. For example, when creating an architecture, an architect will identify applications, "data entities" held within applications, and technologies that implement those applications. These applications will in turn support particular groups of business user or actor, and will be used to fulfil "business services",." 
This usage is more akin to the "atomic elements" of the Zachman framework [8]. Our usage will be more aligned with the capability based definition. We chose to refer to the higher level capability based building blocks which are independent of technology and implementation choices as Business Building Blocks (BBBs) while we use a similar approach to TOGAF for Solution Building Blocks (SBBs ). The former relate to capabilities that the business requires. They can encapsulate services, functionality, data and user access requirements. The latter relate to components chosen to meet the needs of requirements identified in BBBs. They will typically represent commercial off the shelf systems (COTS), data sources or key technologies.

The workshop on BBBs resulted in a whiteboard / Powerpoint model shown as Figure 2. Boxes denote Business Building Blocks i.e. capability; green arrows denote dependencies; dashed arrows denote events; green bubbles are release numbers and yellow text blocks are building block idendities; Generic support capabilities were normalised from the initial diagram and are shown at the base as horizontal lines; User interface modes are shown across the top as horizontal lines. Looking at the figure, we can see that Release 1 would comprise: a web interface; Product Definition (for a limited product set); Price Calculation and an Audit Trail. Release 2 would add: Product Definition for additional products; Workflow and Event handling; Document composition.

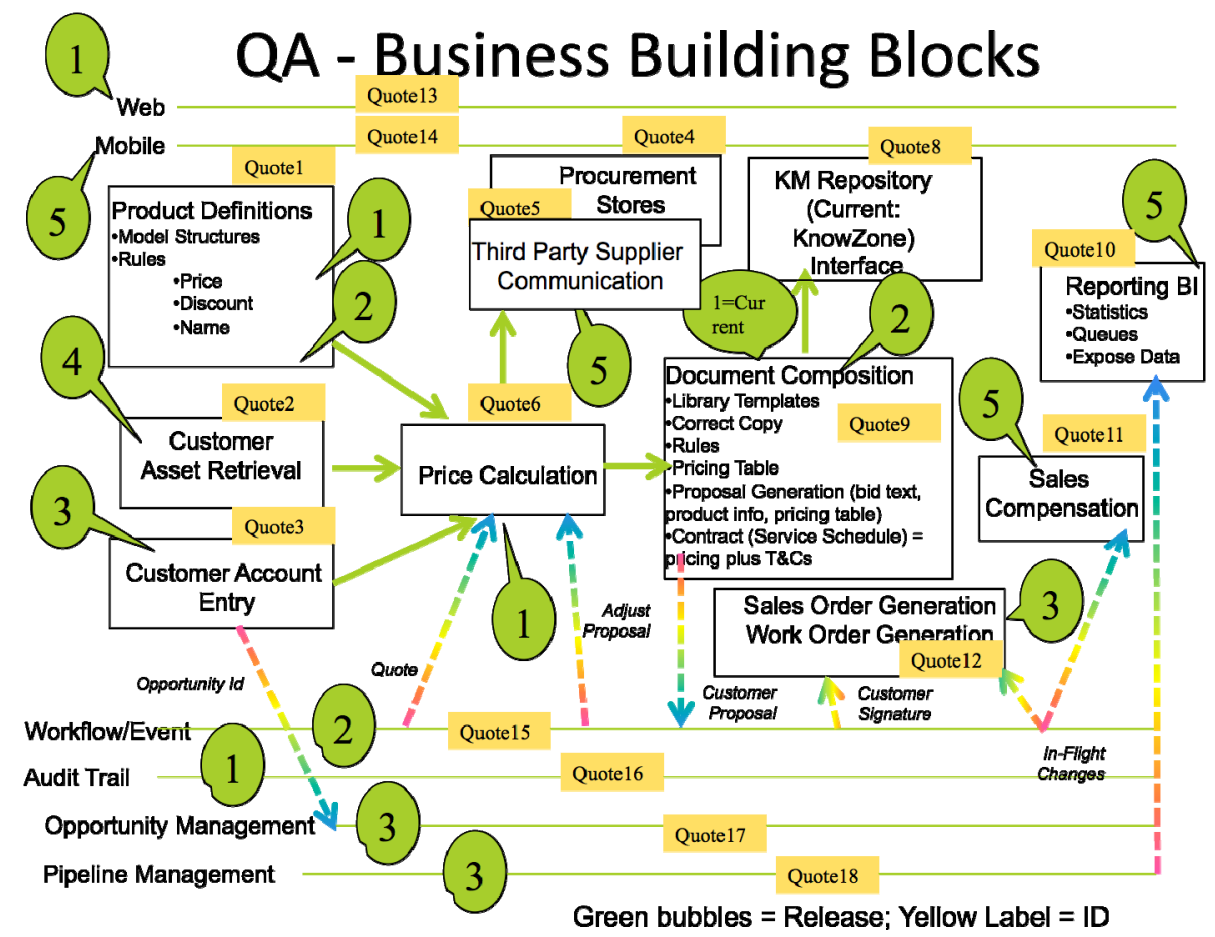

Fig. 2. Business Building Blocks for Quoting 
We standardised terminology to describe a release as a package of capability that would be delivered to the business. Versions may be used by the maintenance team within IT to refer to adjustments of a release without major new functionality.

The initial iteration did not have the release annotations (green callout bubbles) or the block ids (yellow text). The initial diagram was validated with the business analyst, the IT project leader and participants from the Billing project who would deal with the results and data downstream. The programme manager, who was also a key player in defining the longer term strategy, participated in identifying dependencies and desirable business capabilities in terms of value. The block ids were subsequently added to provide concise and unambiguous reference as well as support traceability.

A later audit and similar building block definition for the Billing project surfaced common requirements in the Product Management capability. Quoting and Billing projects had been addressing this independently. Other projects in the organization were also finding this a dependency and trying to address shortfalls. Consequently, the EA function initiated a separate Product Management project. Defining the BBBs for Quoting also highlighted the need for a capable document composition solution. This was required in other areas of the business and a project was initiated to investigate this.

Table 1. Release matrix

\begin{tabular}{|c|c|c|c|c|c|c|}
\hline Target Date $=>$ & & $\begin{array}{l}\text { April } \\
2013\end{array}$ & $\begin{array}{l}\text { June } \\
2013\end{array}$ & & & \\
\hline Target Capability V & \begin{tabular}{|l|} 
Systems \\
Interdependenci
\end{tabular} & \begin{tabular}{|l|} 
Release \\
1
\end{tabular} & $\begin{array}{l}\text { Release } \\
2 \\
\end{array}$ & \begin{tabular}{|l|} 
Release \\
3
\end{tabular} & $\begin{array}{l}\text { Release } \\
4\end{array}$ & \begin{tabular}{|l} 
Release \\
5
\end{tabular} \\
\hline Product Definitions & Tribold & EIA & EIA & & & \\
\hline Price Calculation & Tribold & EIA & & & & \\
\hline Audit Trail & & EIA & & & & \\
\hline Document & Qvidian & & EIA & & & \\
\hline Workflow & & & EIA & & & \\
\hline Customer & Siebel & & & EIA & & \\
\hline Sales Order/Work Order & Siebel & & & EIA & & \\
\hline Installs $\quad$ Project & Siebel & & & EIA & & \\
\hline Customer Asset Read & Siebel & & & & EIA & \\
\hline Opportunity Management & Siebel & & & & & \\
\hline Pipeline Management & Siebel & & & & & \\
\hline $\begin{array}{l}\text { Sales Compensation } \\
\text { Calculations }\end{array}$ & $\begin{array}{l}\text { Oracle } \\
\text { Incentive }\end{array}$ & & & & & \\
\hline \multicolumn{7}{|l|}{ Web/Mobile Access } \\
\hline Reporting \& BI & Microstrategy, & & & & & \\
\hline \multicolumn{7}{|l|}{ 3rd Party Communication } \\
\hline \multicolumn{7}{|l|}{ Procurement/Stores } \\
\hline \begin{tabular}{|ll} 
Knowzone & Sales \\
Document Creation & \\
\end{tabular} & Knowzone & & & & & \\
\hline Authority Matrix & & & & & & \\
\hline
\end{tabular}


A further scoping issue was related to content of the delivered solution in terms of product/service types to be addressed per release. The initial release of Quoting would only provide support for one major product. Subsequent releases will add additional products, which in some cases involves not only the product modeling and capture into the solution systems, but additional system and interfacing capability, e.g. to handle "bundles" which are products composed from other offerings. We did not want to complicate the BBB diagram with this dimension, so opted to create a matrix which provides a view of capability, release timing and content coverage. The latter is provided in the cells as either (i) a list of product identities or (ii) sub-capabilities of building blocks (also shown in Figure 1 as bullet points within the block text). This is shown as Table 1.

\section{$4 \quad$ Taking It Further}

Following the development of the BBB diagram, addition of release tagging, and the release content and timing matrix, a meta model was developed to allow repository and tool support for the building block model techniques. The organization uses the EVA Netmodeler web and repository based EA toolset [9]. Customising the meta model and defining suitable visual notation "model types" allows support for the required techniques in a shared repository. The EA team believed this would enhance rigour, promote sharing and provide visibility across projects. The resultant meta model is shown as Figure 3.

Building Block Categories allow differentiating between business and solution building blocks. Note that multiple own-type relationships exist between building blocks. These are necessary to capture the semantics between building blocks within a layer, as well as between business and solution building blocks. Content elements are related to a building block and a release. They occur in varieties of Feature, Product/Service and Location. These correspond to the cells in the previously presented release matrix (Table 1). The relationship via Model to Value Adding Activity links the building block model to the relevant value chain step.

We were able to define visual model types in the tool to create equivalent business building block diagrams to the Powerpoint model shown earlier. See Figure 4. These do not have the visual cues for release mapping and BBB id but these attributes are captured behind the scenes and can be reported upon or navigated easily.

We also created a visual model type for a solution building block (SBB) diagram. This was completed for the Quoting project, with input from the project team, the business analyst and members of the Billing team who had high knowledge of the existing infrastructure and telecomms processes in general. The resultant SBB model is shown as Figure 5. This shows actual incumbent or anticipated application system components, technologies and data sources. In building this view across the two projects, the need for a messaging infrastructure and consistent data for shared domain objects was apparent. The former was represented in the diagram as an Enterprise Service Bus (ESB) while the latter translates to the Master Data Management (MDM) element. Projects are now underway in the environment to address these requirements. 


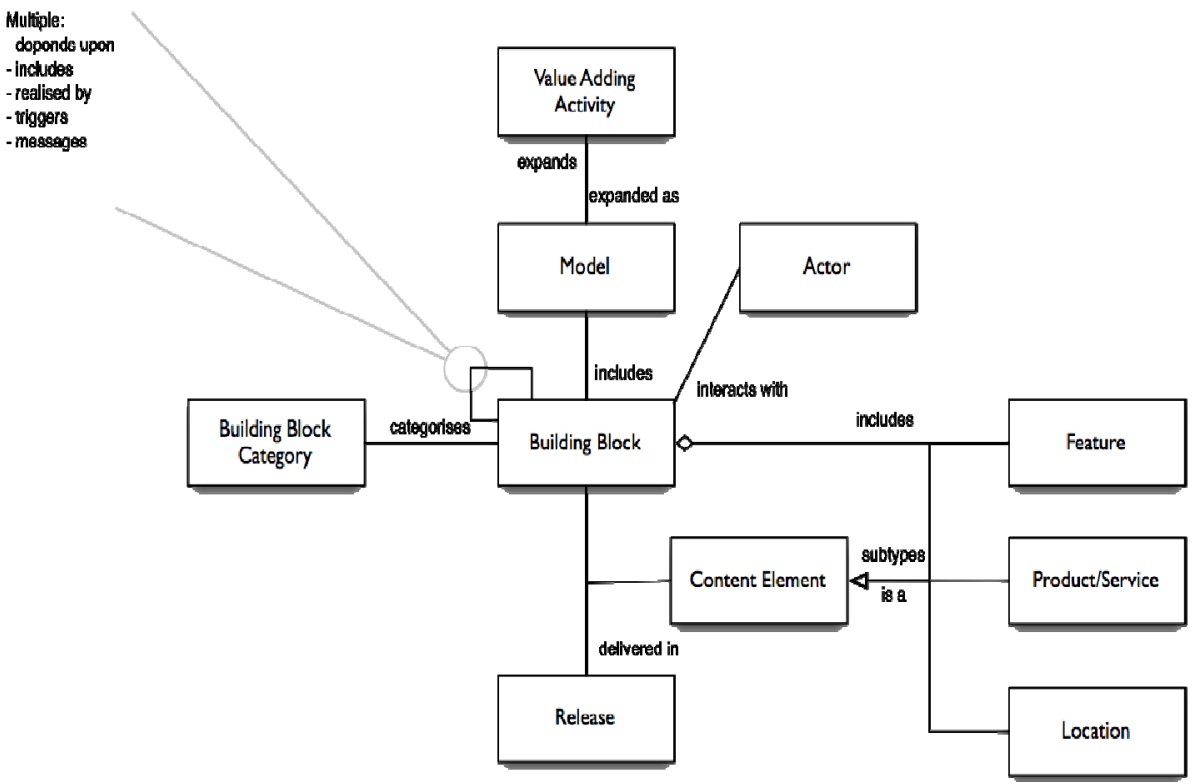

Fig. 3. Meta model for supporting the building block approach

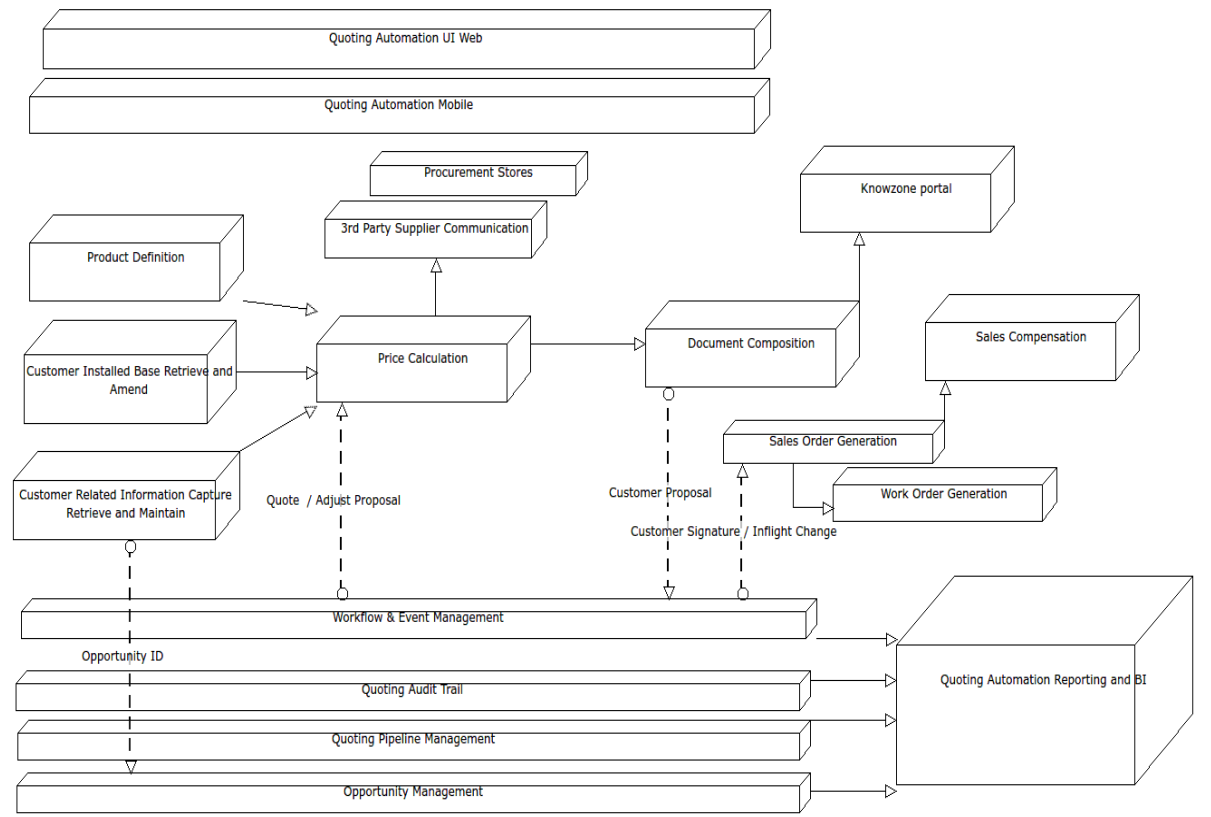

Solid edges: dependency Dotted edges: Data Flow

Fig. 4. Business building blocks model 


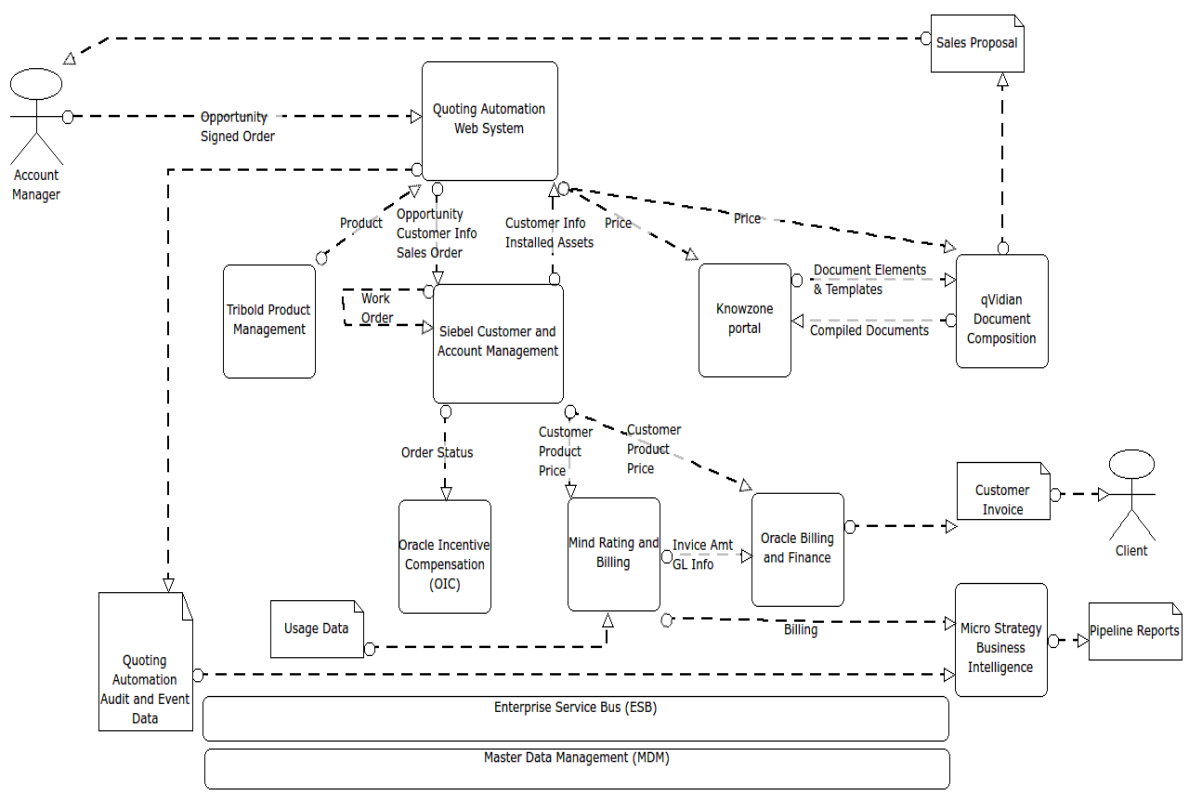

Fig. 5. Solution building blocks model

The revised building block models and the release matrix were presented to stakeholders and the project sponsor in different sessions. Both sessions went extremely well. Stakeholders were easily able to apprehend the business capabilities that were represented and the relationships and dependencies between them. They were also able to grasp the implications of dependencies for release composition. It was clear to them what each release would deliver in terms of functionality and content. With the release matrix, this also relates to delivery time expectations. The three deliverables thus greatly facilitated accurate communication and arriving at a consensus view with stakeholders. Stakeholders expressed their appreciation that they now had a grasp of the scope, capabilities, delivery schedule, release breakdown and dependencies which would enable them to plan properly and engage meaningfully with the development team.

The project sponsor was also delighted with the results, exclaiming that she, for the first time in months, had an accurate and usable picture of what the project was about, what it would deliver, how it was put together and what would be required to deliver it. The programme manager/strategist also expressed her high satisfaction with the clarity which had been achieved.

From an EA perspective we were keen to expand the approach to other projects and to ensure that the clarity prevailed. We thus initiated a number of other activities:

- The business analyst was asked to bring the BBB perspective into the BRS. This was achieved with the BBB ids shown earlier. The BRS was tagged with the relevant ids to show where the requirements mapped onto building blocks. We also encouraged the programme manager and 
business analyst to align the programme management milestones with the $\mathrm{BBB}$ view and to update the project charter to reflect the release plan. In the end they chose to do the alignment in the milestones in the programme management tool, Sciforma [10] and to keep the release map matrix in the BRS.

- The development team was asked to tag the requirements in the agile management tool with the $\mathrm{BB}$ ids, so that we could track progress on development, testing and delivery of BB capabilities.

- $\quad$ The Billing team was engaged to produce similar models for their scope.

- A BBB model was developed for the Product Management capability in the organization. This, together with a phase plan, is now driving improvements in this area

- Other teams (Unified Communications and Data and Information Management) have been engaged and are producing similar models.

Sponsors, stakeholders, EA personnel, programme managers and strategist have all embraced the approach and are very positive about its benefits. The jury is still out on the development group response. They have complied, but we suspect that, for them, there is a bit more overhead than for the other groups, while they see less direct benefit to themselves. There may also be an element of resentment that an oversight group has interfered in a work process with which they were happy. Unfortunately other stakeholders were not satisfied. We hope that over time they will be convinced of the benefits in terms of improved stakeholder communication and a happy sponsor.

To summarise, the benefits include:

- Clear scope of projects via BBBs representing business capabilities and their collation into a picture per project

- Clear tie between value chain and project scope via decomposition of value chain activities into models holding the BBBs supporting that business capability

- Clear release content and planning, via the tagging of the BBBs and the collation of these into releases with delivery times

- Improved communications and agreement between strategy, programme management, sponsor, stakeholders and project team via accessible shared and simple pictures and matrix

- Identification of common requirements at both business and technical levels via visibility in respective building block diagrams

- Common basis for prioritisation within and across projects using the shared definitions of building blocks, dependencies identified and stakeholders knowledge of business issues and benefits

- Simple views at business and technical levels which are not arduous to produce and allow rich discussion and a shared understanding

- Traceability of requirements from business to solution to implementation effort via the linkages created between the building blocks views, the $\mathrm{BRS}$ and the agile requirements management 


\section{$5 \quad$ Limitations}

The experience is reported for one organisation only. The techniques have now been applied to several projects and seem to work equally well across these. Some of these projects are core value chain and targeted at operational improvement while some are more strategic, aimed at business change (e.g. introduction of a new product category). Others, such as Product Management and Data and Information Management are broad supporting capabilities across the value chain. However, all projects are still in the same organisational setting and culture. From our experience in many organisations, industries and EA groups we believe that they will have broad applicability and will work in many settings, but this is no guarantee that they will actually work, or work as well there.

Some of the benefits mentioned, particularly those related to commonality across projects and improved traceability are likely to be reduced, or harder to obtain, if the techniques are not supported by a shared and capable repository.

\section{$6 \quad$ Further Work}

The EA function is working to integrate the approach with business cases and prioritization in the organization. The models will also be used to underpin estimating for capital budgeting purposes.

More work can be done in the area of integration and feedback with the agile management practices used in the development group.

Integration with programme management is underway, but this could be enhanced, and possibly to some extent automated by feeding project, building block and release information across to the programme management tool.

Integration with portfolio management could be investigated for better reuse of information about the existing solution building blocks and infrastructure elements. Full baselines are still being built in the environment and this would require these and a mapping to reference models (e.g. Telecommunications Application Model (TAM), Shared Information/Data (SID) model, eTOM process model [11] from the TM Forum ).

A longitudinal assessment of how the techniques work down the full project lifecycle for requirements traceability should also be undertaken.

\section{References}

1. Porter, M., Millar, V.: How information gives you competitive advantage. Harvard Business Review, 149-174 (July-August 1985)

2. Mcleod, G.: The Inspired Enterprise Architecture Frameworks. Inspired/Promis White Papers, 1-28 (2009)

3. Mcleod, G.: An Inspired Approach to Business Architecture (2012),

http: / /www. inspired.org/busarch/

InspiredApproachToBusinessArchitecture.pdf (retrieved) 
4. The Open Group, TOGAF ® Version 9.1 (2011)

5. Dingsøyr, T., Dybå, T., Moe, N.B. (eds.): Agile Software Development: Current Research and Future Directions. Springer, Heidelberg (2010)

6. Dybå, T., Dingsøyr, T.: Empirical studies of agile software development: A systematic review. Information and Software Technology (2008), doi:10.1016/j.infsof.2008.01.006

7. Vlaanderen, K., Jansen, S., Brinkkemper, S., Jaspers, E.: The agile requirements refinery: Applying SCRUM principles to software product management. Information and Software Technology 53(1), 58-70 (2011), doi:10.1016/j.infsof.2010.08.004

8. Zachman, J., Sowa, J.: Extending and formalizing the framework for information systems architecture. IBM Systems Journal 31(3) (1992)

9. Inspired (2011), EVA Netmodeler, http: / /www. inspired.org/resources / EVA-Brochure-2-6.pdf

10. Sciforma, Sciforma 5.0 Programme Management (2012), http: / /www.sciforma.com

11. Telemanagement Forum (TMForum), FrameWorkx (2013), http: / /www. tmforum.org 